\title{
Noisy logo recognition using line segment Hausdorff distance
}

\author{
Jingying Chen, Maylor K. Leung*, Yongsheng Gao \\ Center for Graphics and Imaging Technology, School of Computer Engineering, Nanyang Technological University, \\ 639798 Nanyang, Singapore
}

Received 18 January 2002; accepted 21 May 2002

\begin{abstract}
Logo recognition is of great interest in the document and shape analysis domain. In order to develop a recognition method that is robust to employ under adverse conditions such as different scale/orientation, broken curves, added noise and occlusion, a modified line segment Hausdorff distance is proposed in this paper. The new approach has the advantage to incorporate structural and spatial information to compute dissimilarity between two sets of line segments rather than two sets of points. The proposed technique has been applied on line segments generated from logos with encouraging results. Clear cut distinction between the correct and incorrect matches has been observed. This suggests a strong potential for logo and shape recognition system.
\end{abstract}

(C) 2002 Pattern Recognition Society. Published by Elsevier Science Ltd. All rights reserved.

Keywords: Hausdorff distance; Line; Logo matching; Shape

\section{Introduction}

Logo recognition is of great interest in the document and shape matching domain. Logos can act as a valuable means in identifying sources of documents. By recognizing the logo, semantic information about the document is obtained which may be useful to decide whether or not to analyze the textual parts. Logos are 2D shapes of varying complexity, with interior and exterior contours that are not necessarily connected. Therefore the recognition process seems to be difficult because of its complexity. Some researchers have investigated the problem of logo recognition, by applying algebraic and differential invariants [1], positive and negative shape feature [2], Zernike moment [3], string-matching technique [4-6], template matching [7], combined measure [8] and interactive feedback [9]. Although some very effective results have been found for clean logos assuming good image quality and perfect segmentation of logos from the document, they can hardly be robust with corrupted logos, such as strips obstructing the logo in unpredictable

\footnotetext{
* Corresponding author.

E-mail address: asmkleung@ntu.edu.sg (M.K. Leung).
}

positions. Other research on logo recognition by artificial neural network $[10,11]$ can deal with noisy logos, however, it requires a lot of training time before it can be used. In order to develop a logo recognition method that is robust to employ under noisy condition and produces intuitively reasonable results, a modified line segment Hausdorff distance (LHD) is proposed for logo recognition in this paper. The new approach has the advantage to incorporate structural and spatial information to compute the dissimilarity between two sets of line segments rather than two sets of points. The added information can conceptually provide more and better distinctive capability for recognition. The uses of line segment matching were also adopted in Refs. [12,13]. De and Aeberhard [12] proposed image-based face recognition algorithm that employed a set of line segments to represent 2D face image, together with a nearest-neighbor classifier as the line segment matching scheme. This method is robust to rotations and different scales but not to occlusion. Zhang and Shu [13] employed line segment extraction and line matching to recognize palmprints for personal identification. Although it is simple and efficient, it can hardly be robust to noisy palmprints. In this paper, the proposed LHD has been applied on line segments generated from logos with 


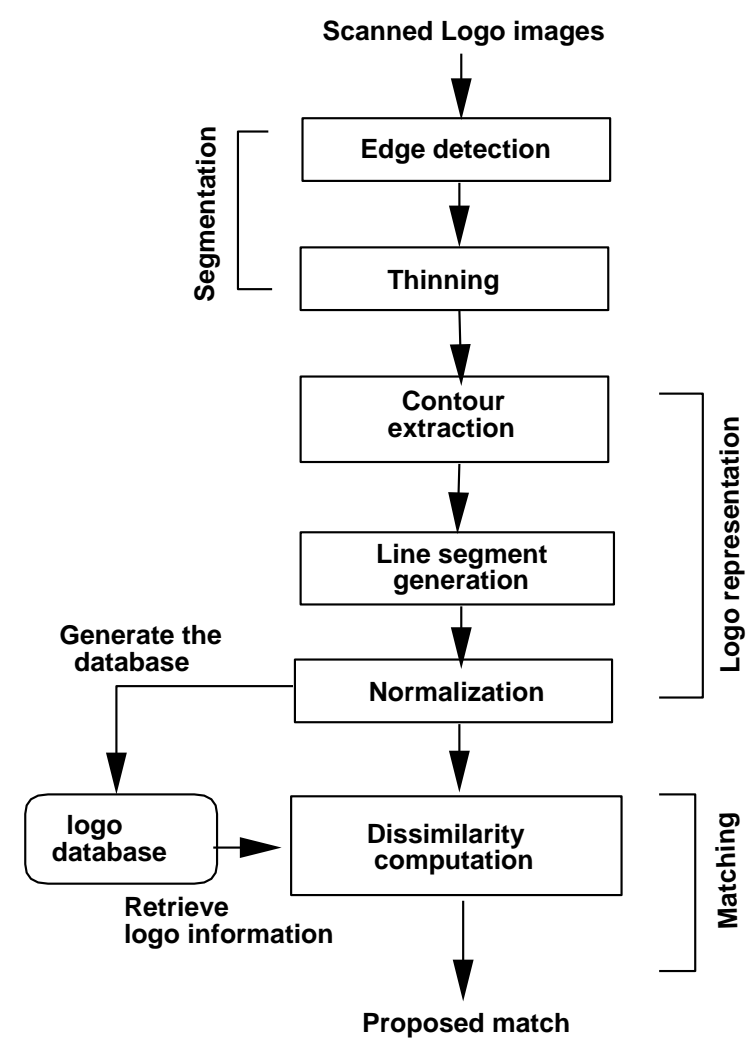

Fig. 1. The proposed system flowchart of logo recognition.

encouraging results. Clear cut distinction between the correct and incorrect matches has been observed. This suggests a strong potential for logo and shape recognition system.

The outline of the paper is as follows. A system overview is described in Section 2. Section 3 reviews the Hausdorff distance. A modified LHD is presented in Section 4. Section 5 describes the results while Section 6 presents the conclusions and directions for future work.

\section{System overview}

The proposed logo recognition system comprises three modules, i.e. segmentation, logo representation and matching. The segmentation operates on raw input of the logo images (i.e. test images). Outputs of matches are dissimilarity distances between models and test images. The system flowchart is illustrated in Fig. 1, where each process will be described briefly in the following.

\subsection{Segmentation}

After scanning, the edge detection and thinning processes are performed to segment a logo. Edge detection is to detect discontinuities in the image intensity. The Nevatia edge detector [14] is used in this study. After edge detection, a thinning process is used to reduce thick lines to chains of single pixels that can be easily traversed. Examples of the edge image and thinned image are shown in Fig. 2.

\subsection{Logo representation}

\subsubsection{Contour extraction}

The logo can be further reduced to its contour representation before feeding to the next process. Contour is a compact way to represent the shape of an image. It stores sequential lists of pixel locations which can be represented by integer coordinates. Given a sequence of integer coordinate points $p_{1}\left(x_{1}, y_{1}\right), p_{2}\left(x_{2}, y_{2}\right), \ldots, p_{n}\left(x_{n}, y_{n}\right)$, where $p_{i}$ is a neighbor of $p_{i+1}, 1 \leqslant i \leqslant n$, we have $\left|x_{i}-x_{i+1}\right|$ and $\left|y_{i}-y_{i+1}\right|$ both less than or equal to one but not both equal zero.

\subsubsection{Line segment generation}

The line segment generation [15] is responsible for extracting feature points from the contours and connecting any two consecutive feature points by one line segment to represent the image. The shape of an object can thus be represented in a more compact way and computation time can be saved [16,17]. Fig. 3 illustrates one sample of the line segment representation image.

\subsubsection{Normalization}

Afterwards, normalization is necessary. In this phase, in order to deal with the problem of occlusion and corruption, multiple reference lines of the models or test images have to be selected, i.e. each model or test image will have multiple representations, one for each distinctive reference line. Each representation is generated by transforming the correspondent reference line to a standard location with zero degree orientation and one unit line length. Obviously, the model or test image will be transformed and scaled together with the reference line. Fig. 4 displays a test image and its normalized line segment drawings. Each reference line has been highlighted.

The main advantages of normalization are that a standard alignment can be obtained and all the models can be pre-aligned to save time. However, in case of severe distortion, i.e. all the distinctive lines have been corrupted in the test image, this approach will not be able to find the reference line correspondence and the recognition process will fail. On the other hand, to the best of our knowledge, other approaches cannot tackle such distorted images too. The proposed approach can work well as long as one suitable reference line can be found while other approaches are not likely to succeed. In future work, more sophisticated alternatives to normalize logo images can be investigated to improve the robustness of this system. Finally, the output of normalization will be sent to the matching module which employs the proposed LHD measure to generate a proposed correct match. 


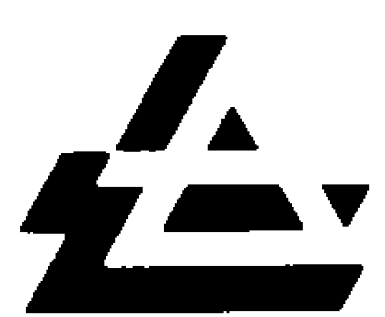

(a)

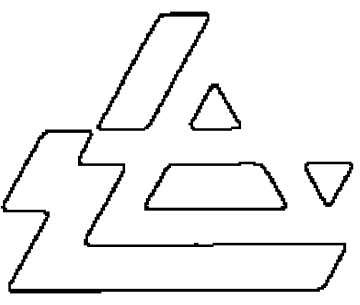

(b)

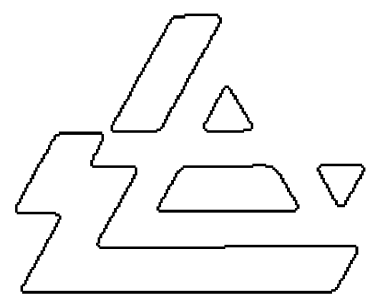

(c)

Fig. 2. An illustration of segmentation: (a) intensity image, (b) edge image, (c) thinned image.

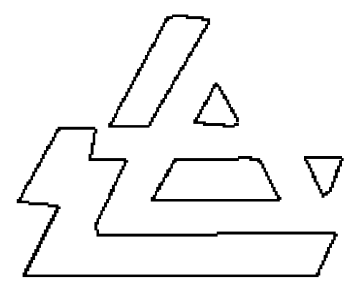

Fig. 3. A sample of the line segment representation image.

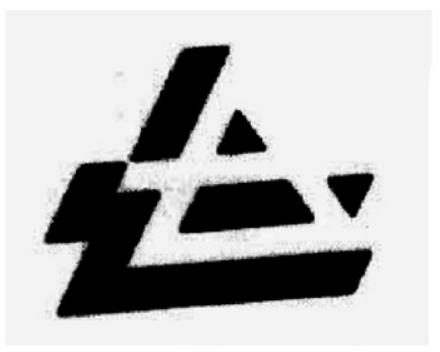

(a)

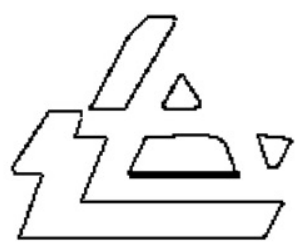

(c)

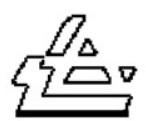

(b)

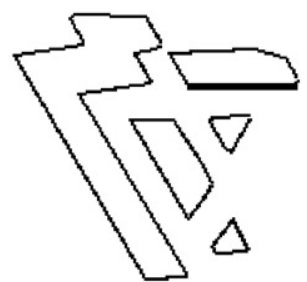

(d)
Fig. 4. One example of a test image (a) and its normalized line segment drawings (b) $-(d)$ according to different reference lines.

\section{Hausdorff distance}

Hausdorff distance is a shape comparison metric based on binary image. It is a distance defined between two sets of points [18]. Unlike most shape comparison methods that build a one-to-one correspondence between a model and a test image, Hausdorff distance can be calculated without explicit point correspondence. Huttenlocher et al. [19] argued that the Hausdorff distance for binary image matching is more tolerant to perturbations on the locations of points than binary correlation techniques since it measures proximity rather than exact superposition.

Given two sets of points $M=\left\{m_{1}, \ldots, m_{p}\right\}$ (representing a model in the database) and $N=\left\{n_{1}, \ldots, n_{q}\right\}$ (representing a test image), the Hausdorff distance is defined as

$H(M, N)=\max (h(M, N), h(N, M))$,

where

$h(M, N)=\max _{m_{i} \in M} \min _{n_{j} \in N}\left\|m_{i}-n_{j}\right\|$

and $\|\cdot\|$ is some underlying distance function for comparing two points $m_{i}$ and $n_{j}$ (e.g. the $L_{2}$ or Euclidean norm). The function $h(M, N)$ is called the directed Hausdorff distance from $M$ to $N$. It identifies the point $m_{i} \in M$ that is the farthest from its nearest neighbors in $N$. Thus the Hausdorff distance, $H(M, N)$, measures the degree of mismatch between two sets. Intuitively, if the Hausdorff distance is $d$, then every point of $M$ must be within a distance $d$ of some point of $N$ and vice versa.

Dubuisson and Jain [20] investigated 24 forms of different Hausdorff distance measures and indicated that a modified Hausdorff distance (MHD) measure had the best performance. The directed MHD is defined as

$h(M, N)=\frac{1}{p} \sum_{m_{i} \in M} \min _{n_{j} \in N}\left\|m_{i}-n_{j}\right\|$,

where $p$ is the number of points in $M$. The definition of undirected MHD is the same as Eq. (1).

The Hausdorff distance defined as Eqs. (1) and (2) is very sensitive to outlier points. A few outlier points, even only a single one, can perturb the distance greatly. The MHD is robust to outlier points that might result from segmentation errors. However, MHD uses spatial information of an image but lacks of local structure representation such as the orientation information. The LHD makes uses of two new attributes of line orientation and line-point association (i.e. points on one line have to match to points on another line but not to points on different lines). In order to provide better distinctive capability for recognition, a modified LHD is proposed here to match logos. The details are described in the next section. 


\section{Modified LHD (MLHD)}

An image object can be abstracted by its boundary or outline that can be represented as a chain of line segments determined by feature points on the boundary. The original LHD measures the distance (dissimilarity) between two line sets, incorporating structural and spatial information. It is built on the distance $d\left(m_{i}, n_{j}\right)$ between two line segments, $m_{i}$ (in the model $M$ ) and $n_{j}$ (in the test image $N$ ) as

$d\left(m_{i}, n_{j}\right)$

$$
=\sqrt{\left(W_{a} \times d_{\theta}\left(m_{i}, n_{j}\right)\right)^{2}+d_{\|}^{2}\left(m_{i}, n_{j}\right)+d_{\perp}^{2}\left(m_{i}, n_{j}\right)},
$$

where $d_{\theta}\left(m_{i}, n_{j}\right), d_{\|}\left(m_{i}, n_{j}\right)$ and $d_{\perp}\left(m_{i}, n_{j}\right)$ are the angle, parallel and perpendicular distances. $W_{a}$ is the weight of angle distance. The LHD measure is based on rather well segmented line segments. Unfortunately, owing to factors such as variations in input devices, geometric distortion, noise, illumination non-uniformities and so on, the consistency and accuracy of feature point detection can be affected. Hence, some error correction mechanisms are needed for LHD. The modified LHD (MLHD) is built on $d\left(m_{i}, \mathscr{N}\right)$ that represents the distance between a model line segment $m_{i}$ and $\mathscr{N}$ (the collection of test image line segments in the neighborhood of $m_{i}$ ) as

$\left[\begin{array}{llll}d_{\theta}\left(m_{i}, \mathscr{N}\right) & d_{\|}\left(m_{i}, \mathscr{N}\right) & d_{\perp}\left(m_{i}, \mathscr{N}\right) & d_{s}\left(m_{i}, \mathscr{N}\right)\end{array}\right]^{\mathrm{T}}$,

where $d_{\theta}\left(m_{i}, \mathscr{N}\right), d_{\|}\left(m_{i}, \mathscr{N}\right), d_{\perp}\left(m_{i}, \mathscr{N}\right)$ and $d_{s}\left(m_{i}, \mathscr{N}\right)$ are angle, parallel, perpendicular and compensation distance.

\subsection{Angle distance}

The angle distance for each $n_{j} \in \mathscr{N}$ is redefined as

$d_{\theta}\left(m_{i}, n_{j}\right)=\min \left(l_{m_{i}}, l_{n_{j}}\right) \times \sin \left(\theta\left(m_{i}, n_{j}\right)\right)$,

where $\theta\left(m_{i}, n_{j}\right)$ computes the smallest positive intersecting angle between lines $m_{i}$ and $n_{j}, l_{m_{i}}$ and $l_{n_{j}}$ denote the lengths of lines $m_{i}$ and $n_{j}$, and $\min \left(l_{m_{i}}, l_{n_{j}}\right) \times \sin \left(\theta\left(m_{i}, n_{j}\right)\right)$ transforms the angular difference into distance as shown in Fig. 5. The scaling constant $W_{a}$ in the original LHD is therefore removed. This modification is more objective and reasonable since one need not select $W_{a}$ to compute $d\left(m_{i}, n_{j}\right)$.

Finally, $d_{\theta}\left(m_{i}, \mathscr{N}\right)$ is defined as

$d_{\theta}\left(m_{i}, \mathscr{N}\right)=\sum_{j}^{J} d_{\theta}\left(m_{i}, n_{j}\right)$

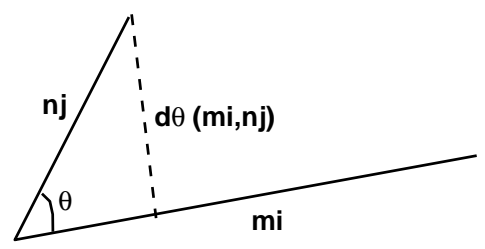

Fig. 5. The illustration of angle distance.

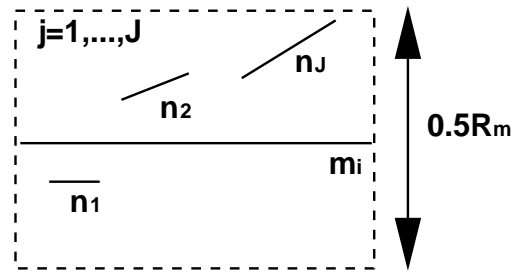

Fig. 6. The neighborhood of $m_{i}$ as: $0.5 R_{m} \times l_{m_{i}}$.

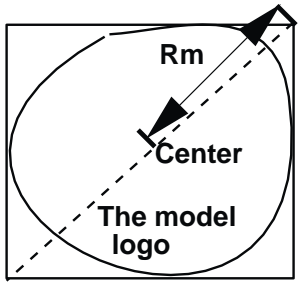

Fig. 7. The computation of $R_{m}$.

to cater for broken lines corresponding to $m_{i}$ in $\mathscr{N} . n_{j}$ is sorted in ascending order according to its perpendicular distance to $m_{i}$ (see Fig. 6), and $J$ is the largest positive (non-zero) number such that the accumulated value of $l_{n_{j}}$ is less than or equal to $l_{m_{i}}$. The minimal value of $J$ is one and $R_{m}$ denotes the size of the normalized model as illustrated in Fig. 7.

\subsection{Perpendicular and parallel distance}

The perpendicular distance for each $n_{j} \in \mathscr{N}$ is modified as

$d_{\perp}\left(m_{i}, n_{j}\right)= \begin{cases}l_{\perp} & \text { if } l_{n_{j}} \geqslant l_{m_{i}}, \\ \frac{l_{n_{j}}}{l_{m_{i}}} \times l_{\perp} & \text { otherwise }\end{cases}$

while the parallel distance remains unchanged as

$d_{\|}\left(m_{i}, n_{j}\right)=\min \left(l_{\| 1}, l_{\| 2}\right)$.

The designs of $\min \left(l_{\| 1}, l_{\| 2}\right), l_{\perp}$ and $d_{\perp}\left(m_{i}, n_{j}\right)$ are illustrated with simplified examples of parallel lines in Figs. 8 and $9 . \min \left(l_{\| 1}, l_{\| 2}\right)$ is defined as the minimum of the displacements to align either the left end points or the right end points of the lines. $l_{\perp}$ is simply the distance between the lines. In general, $m_{i}$ and $n_{j}$ would not be parallel but one can rotate the shorter line with its midpoint as rotation center to the desirable orientation before computing $\min \left(l_{\| 1}, l_{\| 2}\right)$ and $l_{\perp}$. Similarly, $d_{\|}\left(m_{i}, \mathscr{N}\right)$ and $d_{\perp}\left(m_{i}, \mathscr{N}\right)$ are defined as

$d_{\|}\left(m_{i}, \mathscr{N}\right)=\min _{j}^{J}\left(d_{\|}\left(m_{i}, n_{j}\right)\right)$,

$d_{\perp}\left(m_{i}, \mathscr{N}\right)=\sum_{j}^{J} d_{\perp}\left(m_{i}, n_{j}\right)$. 


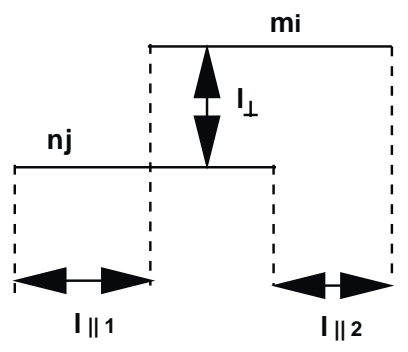

Fig. 8. The line displacement measures.

m

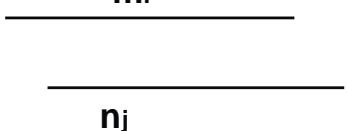

(a) mi

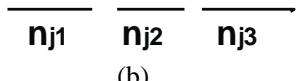

Fig. 9. The illustration of the impact of broken line.

Again, the design is to cater for broken lines. For $d_{\|}\left(m_{i}, \mathscr{N}\right)$, the parallel shifts $l_{\| 1}$ and $l_{\| 2}$ can simply be computed from the left most and right most segments only (e.g. from $n_{j 1}$ and $n_{j 3}$ in Fig. 9(b)); for $d_{\perp}\left(m_{i}, \mathscr{N}\right)$, the weight $l_{n_{j}} / l_{m_{i}}$ is assigned to $l_{\perp}$ such that both Figs. 9(a) and (b) will give the same $d_{\perp}\left(m_{i}, \mathscr{N}\right)$ if $l_{n_{j}}=l_{n_{j_{1}}}+l_{n_{j 2}}+l_{n_{j 3}}$.

\subsection{Compensation distance}

The compensation distance (to cater for $l_{m_{i}}>\sum_{j} l_{n_{j}}$ ) is defined as a non-negative value (i.e. negative value will be reset to zero) as

$d_{s}\left(m_{i}, \mathscr{N}\right)=l_{m_{i}}-\sum_{j}^{J} l_{n_{j}}$.

Finally, $d\left(m_{i}, \mathscr{N}\right)$ and MLHD can be expressed as

$d\left(m_{i}, \mathscr{N}\right)=\sum_{j}^{J}\left[d_{\theta}\left(m_{i}+n_{j}\right)+d_{\perp}\left(m_{i}, n_{j}\right)\right]$

$$
\begin{array}{r}
+\min _{j}^{J}\left(d_{\|}\left(m_{i}, n_{j}\right)\right)+d_{s}\left(m_{i}, \mathscr{N}\right), \\
h(M, N)=\frac{1}{R_{m}} \frac{1}{\sum_{m_{i} \in M} l_{m_{i}}} \sum_{m_{i} \in M} l_{m_{i}} d\left(m_{i}, \mathscr{N}\right),
\end{array}
$$

where the denominator of $1 / \sum_{m_{i} \in M} l_{m_{i}}$ is used to normalize each model logo with its total line segment lengths. Since longer lines are more reliable, the dissimilarity caused by a longer line should create more damages. Based on this rationale, each $d\left(m_{i}, \mathscr{N}\right)$ is multiplied with $l_{m_{i}}$. Finally, the computation is multiplied with $1 / R_{m}$ in order to make the result scale independent. Besides Eqs. (5)-(13), we have also experimented with other formulas. We find that the current setting is derived from intuitive idea and provides better distinctions between the correct and incorrect matches according to our experimental results (see Figs. 13 and 14).

\section{Experimental results}

A series of experiments were carried out using a logo database from the University of Maryland. ${ }^{1}$ It contains 105 distinct logo images in the TIFF format. In this study, 189 test logos, mostly generated from 20 of the 105 models, were used to test the system performance. The $20 \operatorname{logos}$ (see Fig. 10) were selected evenly according to the number of lines detected on each model logo. In order to investigate the effectiveness of logo matching (i.e. whether the test image can be correctly matched to the model or not) and the sensitivity of the system to varying degrees of corruption, we use two groups of test images. In the first group, five types of test images were generated with different types of distortion and noise as shown in Fig. 11. The first set of test images was obtained by rescanning. The test logos differ from the model logos in term of scaling, orientation and random noise. The second set of test images was generated by adding 2 to 3 black and white strips. The strips change the topology of the logo. The third set of test images was generated by cutting the original images. Each cut was less than $\frac{1}{4}$ of the logo size except one cut was almost half of its size. The fourth set of test images was obtained by adding more than one type of noise and distortion, i.e. spot and white Gaussian noise (the spot radius is less than $\frac{1}{4}$ of the image width and standard deviation of Gaussian noise varies from 5 to 45). The last set of test images was scanned from local magazines or downloaded from internet ${ }^{2}$ to get logos foreign to the model database. In the second group, three types of test images were generated and labeled with varying degrees of corruption as shown in Fig. 12. The first set of 36 test images was generated by adding one black strip on six model images (the strip width varies from 5 to 105 pixels). The second set of test images was generated by adding white Gaussian noise on six model images (the standard deviation of Gaussian noise varies from 5 to 85 ). The third set of 18 test images was created by skewing three model images from $5^{\circ}$ to $30^{\circ}$ in the horizontal direction [4].

\subsection{Matching results}

The first test set of 20 regenerated logos has been correctly recognized by the proposed system. The dissimilarities of the correct matches (DCM), versus the nearest incorrect matches (DNIM) of MLHD, are plotted in Fig. 13(I). The test logo names in the $X$-axis direction are sorted according to the digits. Obvious distinctions between DCM and DNIM can be observed. Compared with the original LHD

\footnotetext{
${ }^{1}$ http://document.cfar.umd.edu/pub/contrib/databases/umdlogo database.tar

${ }^{2}$ http://www.hockeydb.com/ihdb/logos
} 


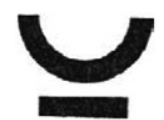

$\log 0105$

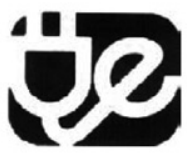

$\log 021$

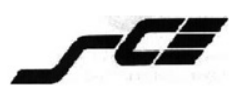

$\log 026$

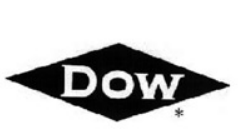

$\log 042$

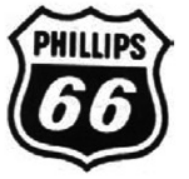

$\log 050$

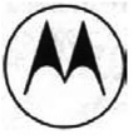

logo11

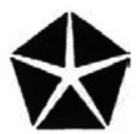

$\log 022$

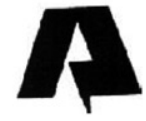

$\log 035$

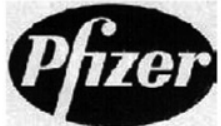

$\log 045$

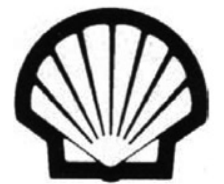

$\log 060$

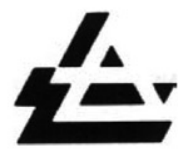

$\log 012$

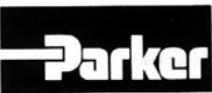

$\log 024$

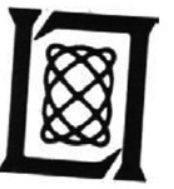

$\log 04$

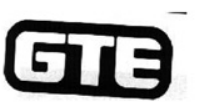

$\log 046$

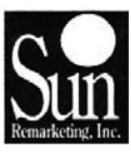

$\log 068$

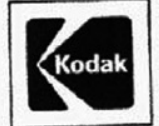

$\log 015$

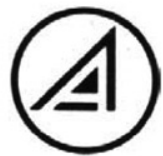

$\log 025$

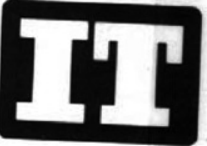

$\log 040$

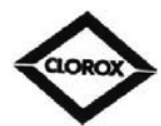

$\log 047$

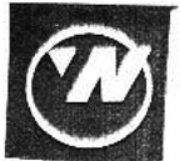

$\log 096$

Fig. 10. The 20 model logos selected to generate the test patterns.

(Figs. 13(II)) and MHD (Fig. 13(III)), larger distinction between the average dissimilarity of the correct matches and the nearest incorrect matches has been observed. Overlapping of projections on the $Y$-axis between DCM and DNIM can be observed in Figs. 13(II) and (III) but not in Fig. 13(I). Hence, it is not possible to find a simple threshold to separate the correct matches from the nearest incorrect matches for Figs. 13(II) and (III). The data suggest that MLHD has better distinctive capability for logo and shape recognition than LHD and MHD. The curves of DCM versus DNIM for two-black-strip and three-white-strip (each strip width was less than 25 pixels), occlusion and mixed noise (the standard deviation of the white Gaussian noise was less than 45) corrupted images are plotted in Fig. 14. From Figs. 14(I), (II) and (IV), one can see that obvious distinctions between the correct and the nearest incorrect matches still persist, all the two-black-strip, three-white-strip and mixed noise corrupted test logos have been correctly matched. From Fig. 14(III), one can observe that the distinctions between DCM and DNIM decrease. This occurred to test logo with occlusion of almost half of its size.
To test behavior on logos not in the downloaded database, foreign logos were fed to the system and the result of the lowest dissimilarities is shown in Fig. 15. It can be observed that such dissimilarities are much higher and can be distinguished from the correct matches shown in Figs. 13 and 14.

\subsubsection{Timing issue}

The timing required for matching logos to the database varies with the numbers of line segments. Logos with more line segments will take longer time as shown in Fig. 16. In future work, shape indexing and retrieval methods employing angular information of the reference lines and global orientations of line segments can be investigated to cut down the processing time.

\subsection{Sensitivity analysis}

In order to assess the behavior of the proposed method under varying degrees of corruption, Fig. 17 records the experimental results for the strip corrupted, white 


\begin{tabular}{|c|c|c|}
\hline Test types & $\begin{array}{c}\text { Number of } \\
\text { test logos }\end{array}$ & Test examples \\
\hline $\begin{array}{c}\text { Regenerated } \\
\text { logos }\end{array}$ & 20 & \\
\hline $\begin{array}{c}\text { Strip } \\
\text { corrupted } \\
\text { logos }\end{array}$ & 40 & \\
\hline $\begin{array}{c}\text { Partially } \\
\text { occluded } \\
\text { logos }\end{array}$ & 20 & \\
\hline $\begin{array}{c}\text { Mixed noise } \\
\text { corrupted } \\
\text { logos }\end{array}$ & 20 & \\
\hline $\begin{array}{c}\text { Foreign } \\
\text { logos }\end{array}$ & 5
\end{tabular}

Fig. 11. Examples of the first group of test images.

\begin{tabular}{|c|c|c|c|}
\hline Test types & $\begin{array}{l}\text { Number of } \\
\text { test logos }\end{array}$ & Model logos & Test examples \\
\hline $\begin{array}{l}\text { Strip } \\
\text { corrupted } \\
\text { logos }\end{array}$ & 36 & & \\
\hline $\begin{array}{l}\text { Gaussian } \\
\text { noise } \\
\text { corrupted } \\
\text { logos }\end{array}$ & 30 & & , \\
\hline $\begin{array}{l}\text { Skewed } \\
\text { logos }\end{array}$ & 18 & & \\
\hline
\end{tabular}

Fig. 12. Examples of the second group of test images.

Gaussian noise corrupted and skewed images with varying strip widths, standard deviations of Gaussian noise and skew angles. With increasing corruption, the curves of DCM display an expected upward trend from left to right while the curves of DNIM stay relatively unchanged. It can be seen that the distinctions between DCM and DNIM diminish with larger strip widths, standard deviations and skew angles. These experiments show that the proposed technique degrades elegantly with good tolerances of different types of deformations. 


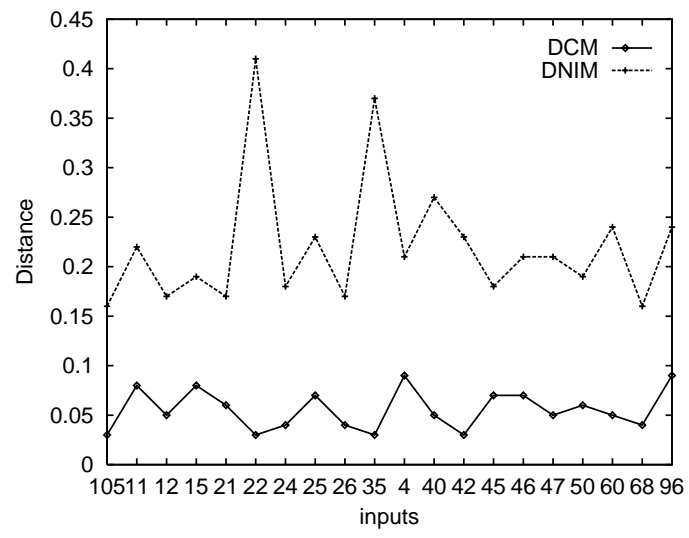

(I)

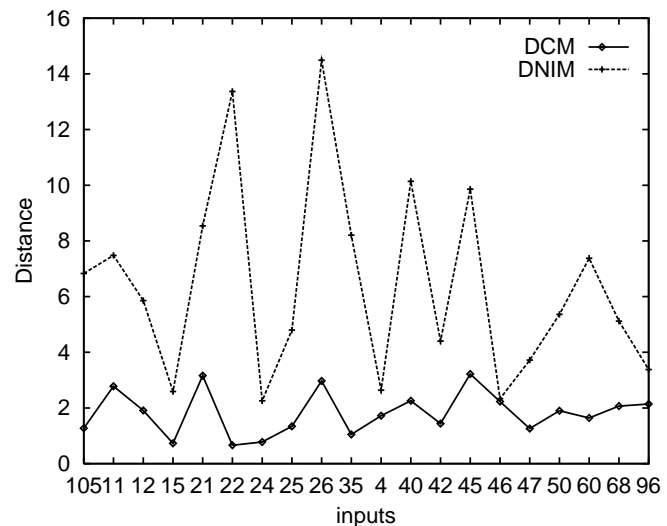

(II)

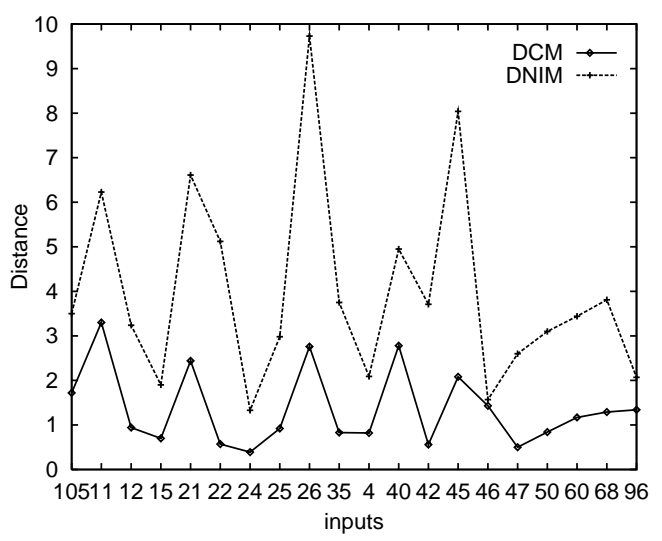

(III)

Fig. 13. Comparison of MLHD, LHD and MHD on regenerated test logos. (I) MLHD, (II) LHD, (III) MHD.

\subsection{Results analysis with respect to $L H D$ and $M H D$}

Excluding the results from foreign logos and severe corruptions (i.e. strip corrupted logos with strip width larger than 25 pixels, white Gaussian noise corrupted logos with standard deviation larger than 45 and skewed logos with skew angle larger than $25^{\circ}$ ), the combined histogram distributions of DCM and DNIM are plotted and compared in Fig. 18. It can be seen that the smallest overlap of distributions occurs for MLHD. Table 1 illustrates the different recognition rates, false positive (FP) and false negative (FN) rates with respect to different threshold values. It can be seen that MLHD scores the best with recognition rate of $99 \%$. From the above experimental results, it is observed that

- Most test logos (99\%) have been classified correctly.

- Using Eqs. (5)-(13), clear cut distinction between the correct and nearest incorrect matches exists (see Figs. 13 and 14). This suggests a simple approach can be used to build a recognition system by thresholding the computed MLHD.
- The proposed technique is robust to noise and distortion. This can be attributed to the use of low level but reliable feature of line segments generated from edge image, i.e. the computation is not dependent on perfect segmentation result like other approaches.

All these positive observations do suggest a strong potential to put the proposed technique into practical use.

\subsection{Discussion and comparison with other methods}

It is difficult to compare the proposed technique with other methods since different approaches have different strengths and weaknesses, and hence each test data set was designed to highlight capability of a particular system. However, to the best of our knowledge, we have not found any system that can tackle as many as distortion types as in our experiment and function as good as the proposed technique. Based on the distortion types tested in each method, one can compare approximately the potential of each method as shown in Table 2. From the table, it can be seen that MLHD is 


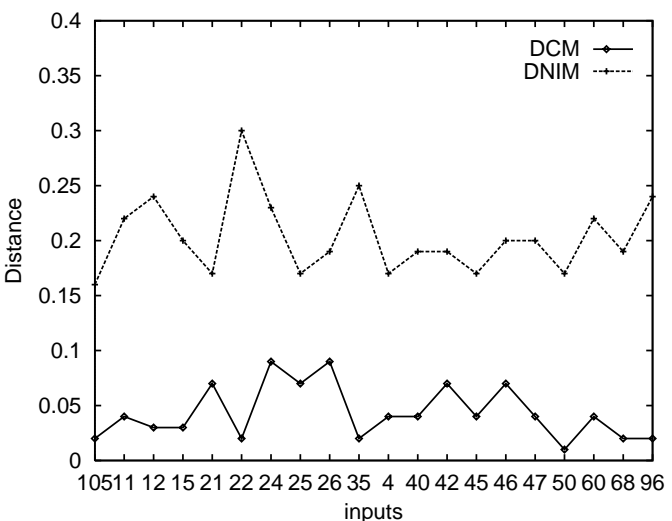

(I)

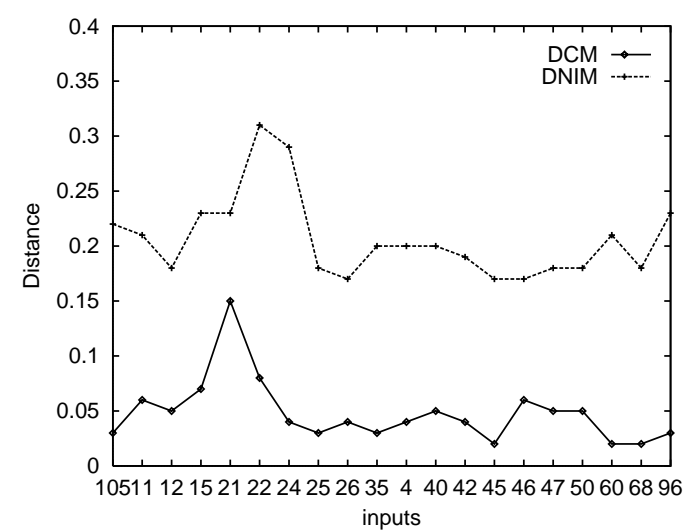

(III)

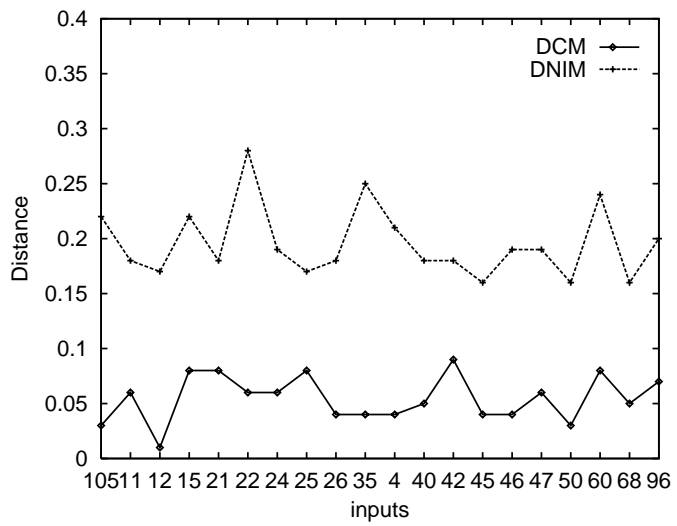

(II)

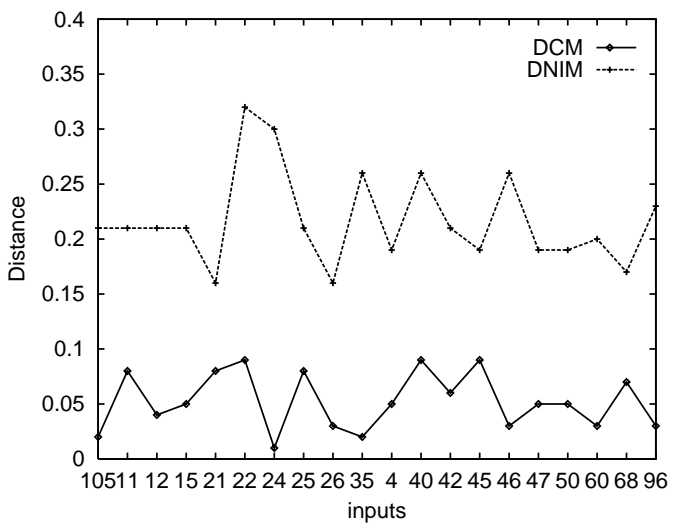

(IV)

Fig. 14. Experiments on strip corrupted, occluded and mixed noise corrupted images. (I) Two-black-strip corrupted, (II) three-white-strip corrupted, (III) occlusion corrupted, (IV) mixed noise corrupted.

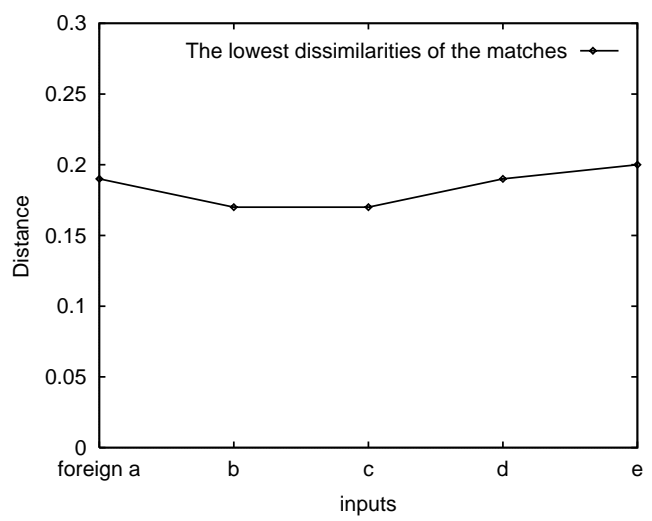

Fig. 15. MLHD measure for five foreign logos.

designed for and can tackle more distortion types. Hence, it scores the best. ${ }^{3}$

\footnotetext{
${ }^{3}$ For comparison purpose, our set of test logos is available by emailing to asmkle ung@ntu.edu.sg.
}

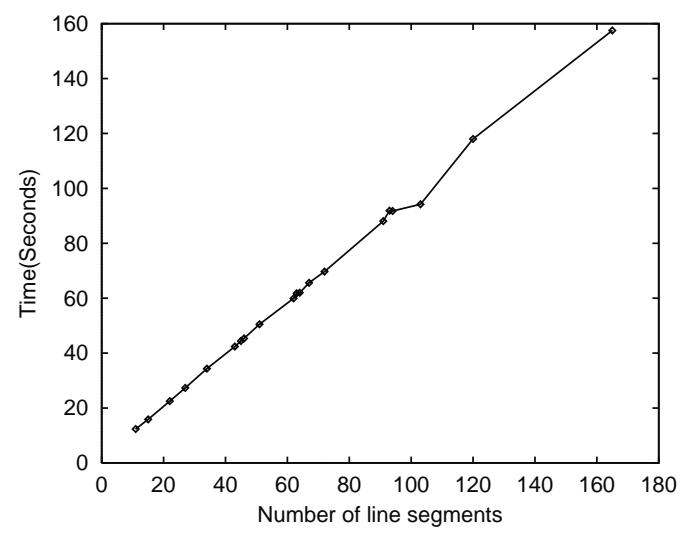

Fig. 16. Average time required for matching one logo with respect to line complexity.

As mentioned in the previous section, MLHD can achieve $99 \%$ recognition rate by setting an appropriate value of the threshold. It can tolerate reasonable amounts of noise and occlusion, and degrade gracefully as these tolerances are 


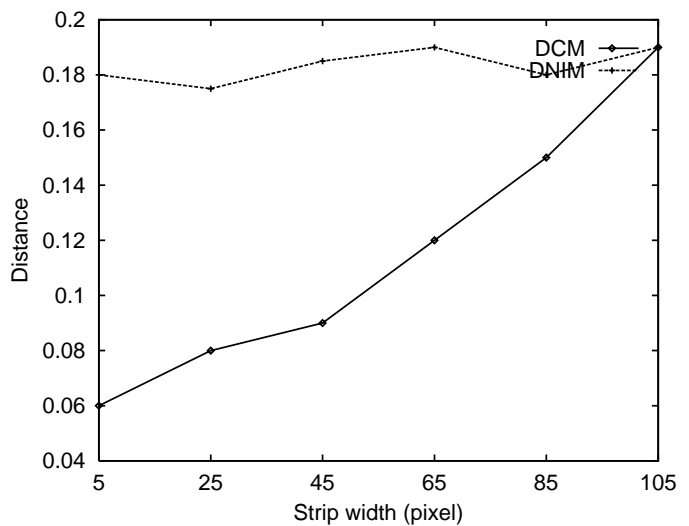

(I)

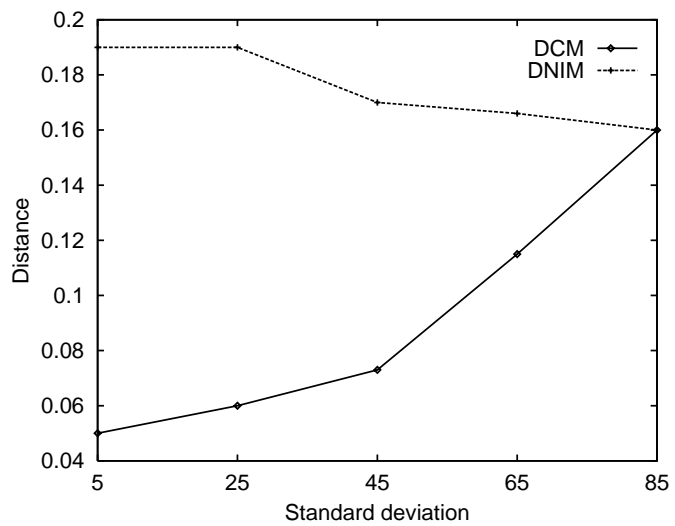

(II)

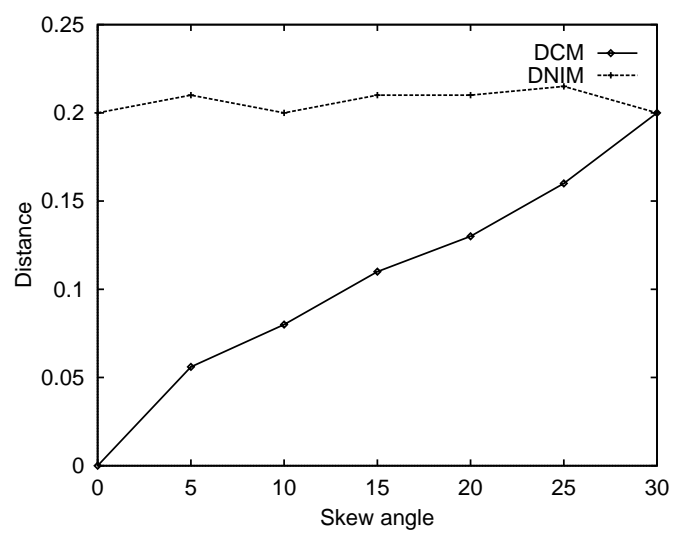

(III)

Fig. 17. Experiments on strip corrupted, white Gaussian noise corrupted and skewed images with different strip widths, standard deviations and skew angles. (I) Strip corrupted, (II) white Gaussian noise corrupted, (III) skewed.

exceeded. In geometric invariant method [1], the experiment was conducted using rotated and scaled logos from a database of approximately 100 logos. The correctly matched logo is among the top three matches. By using positive and negative shape feature [2], Soffer and Samet tested their system performance based on the database of 130 logos. Although edited logos (generated by adding, removing or skewing components that make up the logos) can be recognized, the system cannot handle structural changes, such as a noisy line connecting components of a logo. Kim and Kim [3] employed the Zernike moments to extract 'visually salient feature' that dominantly affects the global shape of trademarks by ignoring minor details. Their method depends on radial complexity and $\mathrm{m}$-fold circular symmetry of the shape. The authors examined the proposed system with ten test trademarks and a database of 3000 trademarks. Among the top 30 retrieved trademarks, the correct rate is $92.5 \%$. This method is efficient because the computation complexity for moment is low, and it can deal with certain noise that does not change the global structure of the shape. However, such method based on moments cannot tackle occlusion or shift of its center of gravity. Twan [4] employed string to match skewed logos to models and achieved recognition rate from $100 \%$ to $72 \%$ for the logos with different skew angles from 2 to $45^{\circ}$. Our proposed technique achieves $100 \%$ recognition rate for skew angles below $30^{\circ}$. Jain and Vailaya [7] indexed trademarks using edge direction and invariant moments to retrieve likely candidates of a test trademark. A deformable template matching process was then applied to propose the final match. The experiment was conducted on a database of 1100 trademarks. The test trademarks include hand drawn and filled trademarks. For the indexing stage, the trademark can be correctly retrieved within the top 20 matches with $99 \%$ correct rate under rotated, scaled and added uniform noise $(5 \%)$ conditions. For the matching stage, each input can be correctly identified as the top match. This system has shown good results on whole shape images, however, it can hardly be robust to occluded images. Mehtre et al. [8] indexed and matched trademark database using combined measures (invariant moment, chain coded string, Fourier descriptor, etc.). They tested their system performance on a database of 500 trademarks and achieved $93.8 \%$ 


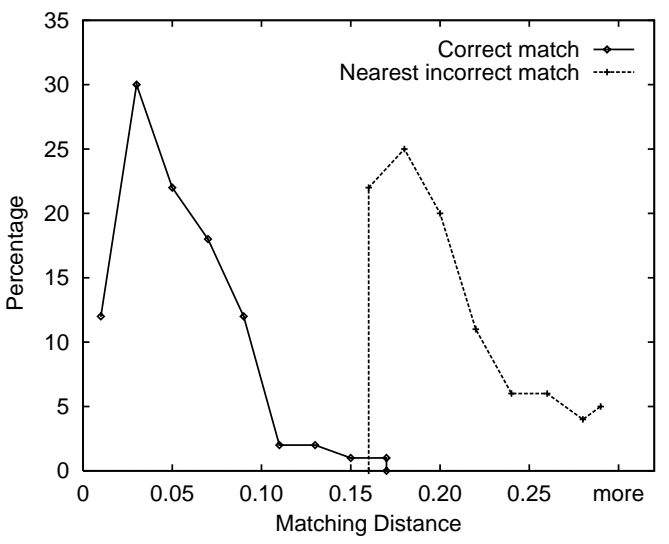

(I)

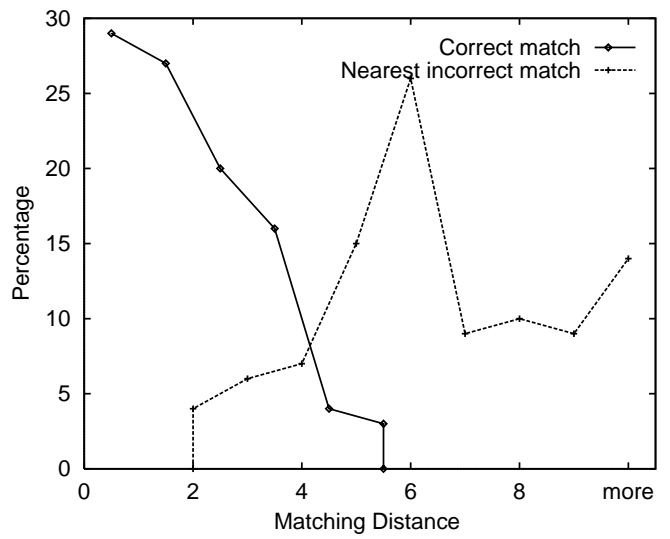

(II)

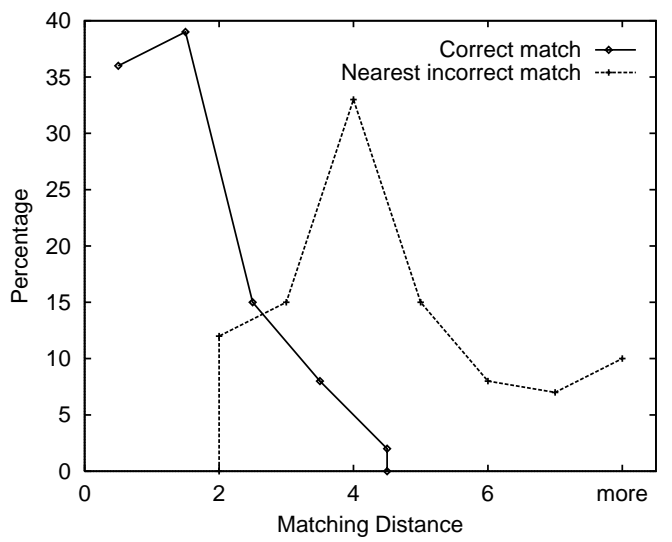

(III)

Fig. 18. Distributions of correct and the nearest incorrect matching distances; vertical axis represents distribution of matching distances in percentage. (I) MLHD, (II) LHD, (III) MHD.

correct rate among top five matches. This approach is simple to implement but not robust to occluded and distorted trademarks. Ciocca and Schettini [9] investigated an interactive system which employed feedback mechanism to extract relevant trademarks. In each iteration the user needs to mark retrieved trademarks as relevant or not relevant. Some success was reported on a database of 1100 trademarks. Since this approach employed global features of the whole images, it is efficient but not suitable for occluded and distorted trademark matching. Cesarini et al. [10,11] employed artificial neural network for logo recognition. The work done in Ref. [11] can tackle the problem of spot noise. The authors examined their system performance using a database of 88 logos. The recognition rate decreases from $100 \%$ to $78 \%$ as the added strip width increases from 0 to 48 pixels. On the other hand, the recognition rate decreases from $100 \%$ to $80 \%$ as the radius of added spot increases from 0 to 75 pixels. The main advantage of this approach is that it is significantly robust with respect to spot noise. However, for neural network methods $[10,11]$, a training stage is needed.
Table 1

The results with different threshold values

\begin{tabular}{llcc}
\hline Threshold & Correct (\%) & FP (\%) & FN (\%) \\
\hline$(I)$ MLHD & & & \\
0.16 & 99 & 0 & 1 \\
0.17 & 77 & 22 & 1 \\
0.18 & 78 & & 0 \\
& & & \\
$(I I)$ LHD & & 0 & 43 \\
2 & 57 & 4 & 23 \\
3 & 73 & 10 & 7 \\
4 & 83 & 17 & 3 \\
5 & 80 & 32 & 0 \\
6 & 68 & & \\
& & & 25 \\
$(I I I)$ MHD & 75 & 0 & 10 \\
2 & 78 & 27 & 2 \\
3 & 71 & 60 & 0 \\
4 & 40 & & \\
5 & & & \\
\hline
\end{tabular}


Table 2

Comparison of logo recognizing methods

\begin{tabular}{|c|c|c|c|c|c|c|c|c|c|}
\hline \multirow[t]{2}{*}{ Methods } & \multicolumn{9}{|c|}{ Distortion types } \\
\hline & $\mathrm{SC}$ & RO & $\mathrm{BL}$ & AS & $\mathrm{OC}$ & GN & SK & $\mathrm{CE}$ & PD \\
\hline MLHD & $\sqrt{ }$ & $\sqrt{ }$ & $\sqrt{ }$ & $\sqrt{ }$ & $\sqrt{ }$ & $\sqrt{ }$ & $\sqrt{ }$ & $\times$ & $\times$ \\
\hline Geometric invariant $[1]$ & $\sqrt{ }$ & $\sqrt{ }$ & $x$ & $\times$ & $\times$ & $\times$ & $\times$ & $\times$ & $\times$ \\
\hline Positive and negative shape feature [2] & $\sqrt{ }$ & $x$ & $x$ & $\times$ & $x$ & $\times$ & $\sqrt{ }$ & $\sqrt{ }$ & $x$ \\
\hline Zernike moment [3] & $\sqrt{ }$ & $\sqrt{ }$ & $\sqrt{ }$ & $\times$ & $\times$ & $\sqrt{ }$ & $x$ & $x$ & $\sqrt{ }$ \\
\hline String matching $[4]$ & $\sqrt{ }$ & $\sqrt{ }$ & $\times$ & $\times$ & $\times$ & $\times$ & $\sqrt{ }$ & $\times$ & $\times$ \\
\hline Template matching [7] & $\sqrt{ }$ & $\sqrt{ }$ & $\times$ & $\times$ & $\times$ & $\sqrt{ }$ & $\times$ & $\times$ & $\times$ \\
\hline Combined measure $[8]$ & $\sqrt{ }$ & $\sqrt{ }$ & $\times$ & $\times$ & $\times$ & $\times$ & $\times$ & $\times$ & $\times$ \\
\hline Interactive feedback [9] & $\sqrt{ }$ & $\sqrt{ }$ & $\times$ & $\times$ & $\times$ & $\times$ & $\times$ & $\times$ & $\times$ \\
\hline Artificial neural network [10] & $\sqrt{ }$ & $\sqrt{ }$ & $x$ & $\times$ & $\times$ & $\sqrt{ }$ & $\times$ & $x$ & $x$ \\
\hline Autoassociator-based artificial neural network [11] & $\sqrt{ }$ & $\sqrt{ }$ & $\sqrt{ }$ & $\sqrt{ }$ & $\sqrt{ }$ & $\times$ & $\times$ & $\times$ & $\times$ \\
\hline
\end{tabular}

$\sqrt{ }$ : has been used to tackle such distortion or transformation. SC: scaling, RO: rotation, BL: random broken line, AS: random added strip, OC: occlusion, GN: Gaussian noise, SK: skew, CE: component editing (adding, removing or skewing logo component), PD: photo deformation such as pinch, punch, sphere, twirl, ripple, and diffuse from any photo editing package.

More training examples would lead to better recognition result. Unfortunately, training is a very expensive process. Based on the above discussion, it is obvious that MLHD has a strong potential for noisy logo recognition.

\section{Conclusion}

This paper has described a novel logo recognition method which aims to produce intuitively correct results with robustness. The proposed modified line segment Hausdorff distance for logo recognition makes uses of structural and spatial information and is robust to noise, occlusion and broken lines that might result from segmentation errors. The added information has conceptually provided more and better distinctive capability for recognition. The novel technique has been applied on line segments generated from logos with encouraging results that supports the concept experimentally. Compared with other researches on logo recognition, the proposed technique is simple in concept and can tackle more distortion and transformation types than the other. Future work can employ the technique on other application areas such as fingerprints, iris pattern and human face. It is also possible to restrict the matching mechanism and develop a curve to curve matching concept.

\section{Acknowledgements}

The authors would like to thank CGIT from NTU for providing the experiment facilities.

\section{References}

[1] D. Doermann, E. Rivlin, I. Weiss, Applying algebraic and differential invariants for logo recognition, Mach. Vision Appl. 9 (1996) 73-86.

[2] A. Soffer, H. Samet, Using negative shape features for logo similarity matching, Proceedings of the Fourth International Conference on Pattern Recognition, Brisbane, Australia, 1998, pp. 571-573.

[3] Y.S. Kim, W.Y. Kim, Content-based trademark retrieval system using a visually salient feature, Image Vision Comput. 16 (1998) 931-939.

[4] T.A. Twan, Linear document processing, Ph.D. Thesis, School of Computer Engineering, Nanyang Technological University, 1998.

[5] H.L. Peng, S.Y. Chen, Trademark shape recognition using closed contours, Pattern Recognition Lett. 18 (1997) 791-803.

[6] G. Cortelazzo, G.A. Mian, G. Vezzi, P. Zamperoni, Trademark shapes description by string-matching techniques, Pattern Recognition 27 (8) (1994) 1005-1018.

[7] A.K. Jain, A. Vailaya, Shape-based retrieval: a case study with trademark image database, Pattern Recognition 31 (9) (1998) 1369-1390.

[8] B.M. Mehtre, M.S. Kankanhalli, W.F. Lee, Shape measures for content based image retrieval: a comparison, Inf. Process. Manage. 33 (3) (1997) 319-337.

[9] G. Ciocca, R. Schettini, Content-based similarity retrieval of trademarks using relevance feedback, Pattern Recognition 34 (2001) 1639-1655.

[10] F. Cesarini, M. Gori, S. Marinai, G. Soda, A hybrid system for locating and recognizing low level graphic items, International Conference on Graphic Recognition, Lecture Notes in Computer Science, Vol. 1072, Springer, Berlin, 1996, pp. 135-147.

[11] F. Cesarini, E. Francesconi, M. Gori, S. Marinai, J.Q. Sheng, G. Soda, A neural-based architecture for spot-noisy 
logo recognition, Proceedings of the Fourth International Conference on Document Analysis and Recognition, Ulm, Germany, 1997, pp. 175-179.

[12] O. De, S. Aeberhard, Line-based face recognition under varying pose, IEEE Trans. Pattern Anal. Mach. Intell. 21 (1999) 1081-1088.

[13] D.P. Zhang, W. Shu, Two novel characteristics in palmprint verification datum point invariance and line feature matching, Pattern Recognition 32 (1999) 691-702.

[14] R. Nevatia, K.R. Babu, Linear feature extraction and description, Comput. Graphics Image Process. 13 (1980) 257-269.

[15] M.K. Leung, Y.H. Yang, Dynamic two-strip algorithm in curve fitting, Pattern Recognition 23 (1/2) (1990) 69-79.
[16] B. Huet, E.R. Hancock, Line pattern retrieval using relational histograms, IEEE Trans. Pattern Anal. Mach. Intell. 21 (12) (1999) 1369-1370.

[17] Y.S. Gao, Human face recognition using line edge information, Ph.D. Thesis, School of Computer Engineering, Nanyang Technological University, 2000.

[18] W.J. Rucklidge, Efficiently locating objects using the Hausdorff distance, Int. J. Comput. Vision 24 (1997) 251-270.

[19] D.P. Huttenlocher, G.A. Klandeman, W.J. Rucklidge, Comparing images using the Hausdorff distance, IEEE Trans. Pattern Anal. Mach. Intell. 15 (1993) 850-863.

[20] M.P. Dubuisson, A.K. Jain, A modified Hausdorff distance for object matching, Proceedings of the 12th International Conference on Pattern Recognition, Jerusalem, Israel, 1994, pp. $566-568$.

About the Author-JINGYING CHEN received her B. Eng degree in 1996 and M. Eng Degree in 1998 from Huanzhong University of Science and Technology, PRC. She is currently working towards her Ph.D. at Nanyang Technological University. Her research interests include Image Analysis, Pattern Recognition and Computer Vision.

About the Author-MAYLOR LEUNG received the B.Sc. degree in Physics from the National Taiwan University in 1979, and the B.Sc., M.Sc. and Ph.D. degrees in Computer Science from the University of Saskatchewan, Canada, in 1983, 1985 and 1992, respectively. Currently, Dr. Leung is an Associate Professor with Nanyang Technological University, Singapore. His research interest is in the area of Computer Vision, Pattern Recognition and Image Processing. Particular interest is on improving security using visual information. Some demo works on video surveillance, face recognition and shape analysis can be found in www.ntu.edu.sg/home/asmkleung. 